\title{
The chasm between public health and reproductive research: what history tells us about Zika virus
}

\author{
Irina Burd $^{1,2} \cdot$ Diane Griffin ${ }^{3}$
}

Received: 24 February 2016 / Accepted: 26 February 2016 / Published online: 4 March 2016

(C) Springer Science+Business Media New York 2016

\begin{abstract}
Zika transmission from mother to fetus and its possible sexual transmission have become a media focus in the past months as a major public health concern. While motherto-fetus transmission, fetal neurologic manifestations or sexual transmission have never been documented for this virus before, other viruses that belong to the same family are very well known to reproductive health workers, clinicians, and researchers. As a member of Flaviviridae family, including hepatitis $\mathrm{C}$ and bovine viral diarrhea virus (BVDV), Zika's pathogenesis may have some parallels with these infections which may pose future questions for public health and research. Vertical transmission of hepatitis $\mathrm{C}$ virus from mother to child is known to occur in up to $10 \%$ of pregnancies. BVDV, a member of Pestivirus genus of Flaviviridae family is not known to be transmitted to humans but is known for its vertical transmission in cattle. BVDV infection at different stages of gestation may lead to a spectrum of adverse pregnancy outcomes, including pregnancy loss and neurologic
\end{abstract}

Capsule A widely available test for Zika is urgently needed. If there are any concerns as to whether Zika may be responsible for mother-to-child transmission or whether sexual transmission may be possible, a historic perspective on viruses of the same family answers these public healthrelated questions.

Irina Burd

iburd@jhmi.edu

1 Integrated Research Center for Fetal Medicine, Department of Gynecology and Obstetrics, Johns Hopkins School of Medicine, 600 N Wolfe Street, Phipps 228, Baltimore, MD 21287, USA

2 Department of Neurology, Johns Hopkins School of Medicine, Baltimore, MD, USA

3 Molecular Biology and Immunology, Johns Hopkins School of Public Health, Baltimore, MD, USA manifestations (including deformations such as hydrocephalus and microcephaly) in the offspring. Similar to hepatitis C, which is a virus of Hepacivirus genus, BVDV is capable of persistent infection, meaning that virus may stay in mother and future generations of calves may be infected as well, which may, in turn, result in persistence of infection in offspring. Would this be a case with Zika virus? Along with mother-to-fetus transmission, sexual transmission is a concerning implication for Zika virus. Would woman become a persistent career or male be able to persistently carry virus with its sperm is yet unknown; yet, there is a concern for the reservoir of infection. Animal models of the disease are urgently needed not only to demonstrate the mother-to-fetus transmission and confirm the fetal neurologic manifestations but also to address the effects of virus on life-long host's immunity and reproductive health. Along those lines, women desiring pregnancies who are identified to travel, have a partner traveling to, or living in the areas of Zika infections should be encouraged to have a preconception consultation with maternal-fetal medicine.

Keywords Zika $\cdot$ Mother-to-child transmission $\cdot$ Sexual transmission virus in seminal fluid

Zika virus belongs to Flaviviridae family, and is thus related to other viruses in this family, such as dengue, Japanese encephalitis, and West Nile, causing neurologic manifestations. Zika virus transmission from mother-to-fetus and its possible sexual transmission have become a media focus in the past months as a major public health concern. While mother-tofetus transmission, fetal neurologic manifestations or sexual transmission have not been documented for this virus until recently, history tells us that other viruses that belong to the 
same family are known to reproductive health workers, clinicians, and researchers.

As a member of the Flaviviridae family that also includes hepatitis $\mathrm{C}$ virus and bovine viral diarrhea virus (BVDV), Zika virus pathogenesis may have some parallels with these infections and pose questions for future public health and research. Vertical transmission of hepatitis $\mathrm{C}$ virus, a virus of the Hepacivirus genus, from mother to child is known to occur in up to $10 \%$ of pregnancies. BVDV, a member of the Pestivirus genus, does not infect humans but is known for its vertical transmission in cattle [1-5]. BVDV infection at different stages of gestation may lead to a spectrum of adverse pregnancy outcomes, including pregnancy loss and neurologic manifestations (including deformations such as hydrocephalus and microcephaly) in the offspring. Similar to hepatitis C virus, BVDV can cause persistent infection, meaning that the virus may stay in the mother and future generations of calves may be infected as well, which may, in turn, result in persistence of infection in offspring. This is thought not to be likely with Zika virus, a member of the Flavivirus genus, but there is a need for careful clinical studies.

Along with mother-to-fetus transmission, sexual transmission is a concern for Zika virus. Whether women can become persistent carriers or a male be able to persistently carry virus in the seminal fluid, and become a reservoir of infection is yet unknown. As it was well pointed out in manuscript by
Rowland et al., simple and cost-effective widely available testing is urgently needed. Further research into screening as well as into animal models of the disease will not only allow to better understand the mother-to-fetus transmission and the fetal neurologic manifestations but also to address the life-long effects of virus infection on host immunity and reproductive health. Until more is known, for women desiring to get pregnant, who travel to, have a partner traveling to, or live in areas of Zika virus infections, it would be advisable to have a preconception consultation with maternal-fetal medicine.

\section{References}

1. Agerholm JS, Hewicker-Trautwein M, Peperkamp K, Windsor PA. Virus-induced congenital malformations in cattle. Acta Veterinaria Scandinavica. 2015;57(1):54.

2. Badman RT, Mitchell G, Jones RT, Westbury HA. Association of bovine viral diarrhea virus infection to hydranencephaly and other central nervous system lesions in perinatal calves. Aust Vet J. 1981;57:306-7.

3. Hewicker-Trautwein M, Liess B, Trautwein G. Brain lesions in calves following transplacental infection with bovine-virus diarrhea virus. J Vet Med B. 1995;42:65-77.

4. Peterhans E, Schweizer M. BVDV: a Pestivirus inducing tolerance of the innate immune response. Biologicals. 2013;41(1):39-51.

5. Lanyon SR, Hill FI, Reichel MP, Brownlie J. Bovine viral diarrhoea: pathogenesis and diagnosis. Vet J. 2014;199(2):201-9. 